\title{
Energy Based Limit Cycle Control of Elastically Actuated Robots
}

\author{
Gianluca Garofalo and Christian Ott, Member, IEEE
}

\begin{abstract}
A new control law for elastic joint robots that allows to regulate an energy function of the system to a desired value is presented in this paper. Being able to either remove energy from the system or inject into it, oscillations can be both damped out and induced. The proposed nonlinear dynamic state feedback controller forces the system to evolve on a submanifold of the configuration space. The reduced dynamics of the system and of the controller itself are similar to a single elastic joint, for which an asymptotically stable limit cycle is obtained regulating an energy function to a positive desired value. When the desired value of the energy function is chosen to be zero, then the asymptotically stable limit cycle reduces to an asymptotically stable equilibrium point. In this case the oscillations are damped out and the desired task-space configuration is reached. The design of the controller extensively uses the concept of conditional stability, so that the limit cycle can be designed for a lower dimensional dynamical system, although it will result to be a limit cycle for the whole system.
\end{abstract}

Index Terms-Robotics; Stability of NL systems; Limit cycle control; Nonlinear systems.

\section{INTRODUCTION}

$\mathbf{E}$ LASTIC joint robots have been lately the focus of numerous researchers because of the capability to perform highly dynamical, explosive and cyclic motions. The energy stored in the elastic elements can be exploited to increase performances, although the underactuation problem makes the controller design more challenging. This is why joint elasticity was originally treated as a disturbance of the rigidbody dynamics [1], [2]. However, novel drive concepts like series elastic actuators [3] or variable impedance actuators [4] deliberately introduce elasticity for implementing torque control, increasing physical robustness, or reaching high output velocities.

The focus of our research is to find new and efficient methods to produce periodic motions that can be used in general and in particular for locomotion [5]-[7]. As shown in [8], [9] walking and running can be effectively described as periodic tasks. In these cases it is more important to stay on a prescribed orbit in the state space, rather than following an exact trajectory in time [5], [10]. Therefore, in this paper we consider the problem of orbital stabilization for elastic joint robots without considering strong simplifying hypothesis, like singular perturbation [11]. The method presented here extends

Gianluca Garofalo and Christian Ott are with the Institute of Robotics and Mechatronics, German Aerospace Center (DLR), Wessling, Germany. email: (gianluca.garofalo(at)dlr.de).

Manuscript received September 23, 2015; revised March 10, 2016 ; accepted August 2, 2016.

This research is partly supported by the Initiative and Networking Fund of the Helmholtz Association through a Helmholtz Young Investigators Group (Grant no. VH-NG-808). the results of our previous work [12], in order to be able to produce a limit cycle for a robot with an arbitrary number of elastic joints. The result is achieved using a strategy similar to what we proposed in [6] and which is conceptually sketched in Fig. 1. The idea consists in forcing the system to reach a subset of the state space and then producing a limit cycle by regulating an energy function to a desired value [6]. The problem of orbital stabilization for rigid joint robots has been addressed for example in [6], [13]-[17]. In [13], [14] a passive control action is designed which allows to decouple the motion along a vector field from the remaining motion. The system is then forced to follow an integral curve of this vector field via a passive control law. In case of a closed integral field, the system thus converges to a closed orbit in the configuration space. In [15] additionally a non-passive control action is proposed to achieve regulation of the final velocity along the vector field. In [16], [17] the concepts of virtual constraint and feedback linearization are used to obtain a closed loop system that generates its own periodic stable motion. In [6], as well as in this extension for the elastic joint case, the problem is formulated based on the null space decomposition introduced in [18] and used for nullspace compliance control in [19], [20]. In contrast to [13]-[15], we produce an asymptotically stable limit cycle in the state space by regulating an energy function in a submanifold of the state space. Additionally, compared to [6], [7], here we consider elastically actuated robots instead of rigidly actuated ones. Therefore, we utilize the physical potential energy of the compliant actuation instead of a virtual potential energy introduced by the controller. This suggests that energy efficient motion can be achieved. Compared to [16] we take advantage of the passivity property of the system and do not completely alter the original dynamics of the system through feedback linearization. Moreover, in [16], [17] a different underactuated problem is considered, as compared to this work. There the underactuation is not due to the elastic joints, but to the lack of one input variable in a rigidly actuated robot. As a consequence, in [16], [17] the challenge is to design virtual constraints which render the zero dynamics stable, while we completely separate the problem of producing the limit cycle from the virtual constraints. The latter, are only responsible for the subspace in which the system will oscillate.

Finally, it is worth to notice that the same control law can be used to regulate the robot to a desired task-space configuration setting to zero the desired value of the energy function, without requiring a tracking of a desired nullspace torque [19], [21].

\section{Motivating EXAMPLE}

In this section we illustrate the main idea behind the control laws based on the regulation of an energy function. 


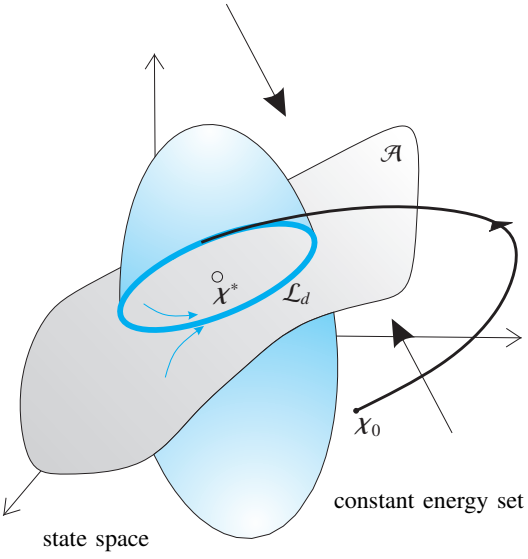

Fig. 1: Conceptual illustration of the main idea. The limit cycle $\mathcal{L}_{d}$ is generated regulating an energy function, after that the system has been forced to evolve on a submanifold $\mathcal{A}$ of the state space.

Consider a simple mass-spring system with dynamic equation given by

$$
m \ddot{q}+k q=u,
$$

with mass $m>0$, stiffness constant $k>0$, state $\chi=(q, \dot{q})$ and input $u$. If we choose $u=0$, the system has an equilibrium point in $\chi^{*}=(0,0)$. For any initial condition $\chi_{0}=\left(q_{0}, \dot{q}_{0}\right)$, the resulting trajectory will be a closed orbit around $\chi^{*}$, that is the level set $\mathcal{L}_{0}$ of the Hamiltonian $H(\chi)=\frac{1}{2}\left(m \dot{q}^{2}+k q^{2}\right)$, defined as $\mathcal{L}_{0}=\left\{\chi \mid H(\chi)=H\left(\chi_{0}\right)\right\}$.

The difference between these closed orbits and limit cycles is that they are not isolated. If we force the system to always reach a desired value of the Hamiltonian $H_{d}>0$, then we will obtain a limit cycle $\mathcal{L}_{d}=\left\{\chi \mid H(\chi)=H_{d}\right\}$. This is possible by choosing $u=-K_{H} \tilde{H}(\chi) \dot{q}$, obtaining the closed loop system

$$
m \ddot{q}+K_{H} \tilde{H}(\chi) \dot{q}+k q=0,
$$

where $K_{H}>0, \tilde{H}(\chi)=H(\chi)-H_{d}$. While in the examined case the set $\mathcal{L}_{d}$ is an isolated closed ${ }^{1}$ orbit in the state space (corresponding to a limit cycle), for higher dimensional systems this is not true and therefore ensuring that the Hamiltonian $H(\chi) \rightarrow H_{d}$ is not enough to conclude the existence of a limit cycle. For this reason, in addition to the regulation of an energy function, in the following we will force the system to evolve on a subset of the state space.

In the remainder of the paper, with an abuse of notation, we will always indicate with $\chi$ the state of the system and with $H(\chi)$ the "energy-like" function which we will regulate to the desired value $H_{d}$, in order to obtain the limit cycle $\mathcal{L}_{d}$, although their definition is different from the one given in this section.

\section{Preliminaries}

\section{A. Model}

In what follows we will consider elastically actuated robots with linear springs in the joints. Assuming to have negligible

\footnotetext{
${ }^{1}$ It is the level set of a positive definite and radially unbounded function.
}

coupling inertias between motor and link side (a typical assumption which is fulfilled in presence of high gear ratios [22]), they can be modeled as

$$
\begin{aligned}
& \boldsymbol{M}(\boldsymbol{q}) \ddot{\boldsymbol{q}}+\boldsymbol{C}(\boldsymbol{q}, \dot{\boldsymbol{q}}) \dot{\boldsymbol{q}}+\boldsymbol{g}(\boldsymbol{q})=\boldsymbol{K}(\boldsymbol{\theta}-\boldsymbol{q}) \\
& \boldsymbol{B} \ddot{\boldsymbol{\theta}}+\boldsymbol{K}(\boldsymbol{\theta}-\boldsymbol{q})=\tau_{m},
\end{aligned}
$$

where $\boldsymbol{\theta}, \boldsymbol{q}, \dot{\boldsymbol{\theta}}, \dot{\boldsymbol{q}} \in \mathbb{R}^{n}$ constitute together the state of the system, being $\boldsymbol{\theta}$ the motor position and $\boldsymbol{q}$ the link position, $\boldsymbol{\tau}_{m} \in \mathbb{R}^{n}$ is the input to the system provided by the motors, $\boldsymbol{M}(\boldsymbol{q}) \in \mathbb{R}^{n \times n}$ is the symmetric positive definite inertia matrix, $\boldsymbol{C}(\boldsymbol{q}, \dot{\boldsymbol{q}}) \in \mathbb{R}^{n \times n}$ is a Coriolis matrix satisfying $\dot{\boldsymbol{M}}=\boldsymbol{C}+\boldsymbol{C}^{T}$, $\boldsymbol{g}(\boldsymbol{q})=\nabla_{\boldsymbol{q}} U_{g}(\boldsymbol{q}) \in \mathbb{R}^{n}$ is the gravity torque vector ${ }^{2}$ and $U_{g}(\boldsymbol{q})$ the gravitational potential. Finally, $\boldsymbol{B} \in \mathbb{R}^{n \times n}$ is the constant, diagonal, and positive definite matrix of motor inertias, $\boldsymbol{K} \in$ $\mathbb{R}^{n \times n}$ is a positive definite diagonal matrix which collects the stiffness constants $K_{i}, i=1, \ldots, n$ of the springs connecting the motors to the links. The total potential energy of the system is given by $U(\boldsymbol{\theta}, \boldsymbol{q})=U_{g}(\boldsymbol{q})+U_{k}(\boldsymbol{\theta}-\boldsymbol{q})$, where $U_{k}(\boldsymbol{\theta}-\boldsymbol{q})$ is the stiffness potential energy.

In case the robot contains not only rotational joints, we will always consider a subset $\mathbb{Q} \subset \mathbb{R}^{n}$ in which all the prismatic joints are kept bounded. In this case it is well known that the following proposition holds [23]

Proposition 1. For every matrix norm there exists a value $\beta$ such that

$$
\left\|\frac{\partial^{2} U_{g}(\boldsymbol{q})}{\partial \boldsymbol{q}^{2}}\right\|<\beta, \forall \boldsymbol{q} \in \mathbb{Q} .
$$

In addition, the following assumption is made:

$$
\beta<\min _{i} K_{i} \quad i=1, \ldots, n,
$$

which, as explained in [19], states that the robot should be designed properly, meaning that the joint stiffness should be sufficiently high to prevent the robot from falling down under the load of its own weight.

\section{B. Coordinate transformation}

We introduce a function $\boldsymbol{x}=\boldsymbol{x}(\boldsymbol{q}), \boldsymbol{x}: \mathbb{R}^{n} \rightarrow \mathbb{R}^{n-1}$, with full rank Jacobian matrix $\boldsymbol{J}(\boldsymbol{q}) \in \mathbb{R}^{(n-1) \times n}$ in order to obtain a 1 dimensional submanifold of the link configuration space defined by $\boldsymbol{x}(\boldsymbol{q})=\mathbf{0}$ [24], as mentioned in the introduction. This allows us to write the dynamics of the system with a new set of coordinates, as in [18], [20]. We first compute a nullspace base matrix ${ }^{3} \boldsymbol{Z}(\boldsymbol{q}) \in \mathbb{R}^{1 \times n}$ to derive a dynamically consistent ${ }^{4}$ nullspace projector $\boldsymbol{N}(\boldsymbol{q})=\left(\boldsymbol{Z}(\boldsymbol{q}) \boldsymbol{M}(\boldsymbol{q}) \boldsymbol{Z}^{T}(\boldsymbol{q})\right)^{-1} \boldsymbol{Z}(\boldsymbol{q}) \boldsymbol{M}(\boldsymbol{q}) \in$ $\mathbb{R}^{1 \times n}$. The latter will be part of the extended Jacobian matrix $\boldsymbol{J}_{N}(\boldsymbol{q}) \in \mathbb{R}^{n \times n}$, such that

$$
\left[\begin{array}{c}
\dot{\boldsymbol{x}} \\
v
\end{array}\right]=\boldsymbol{J}_{N}(\boldsymbol{q}) \dot{\boldsymbol{q}}=\left[\begin{array}{c}
\boldsymbol{J}(\boldsymbol{q}) \\
\boldsymbol{N}(\boldsymbol{q})
\end{array}\right] \dot{\boldsymbol{q}},
$$

${ }^{2}$ With the symbol $\nabla_{x}(\cdot)$ we are indicating $\left(\frac{\partial(\cdot)}{\partial x}\right)^{T}$ in order to ease the notation.

${ }^{3}$ I.e. it fulfills the condition $\boldsymbol{J}(\boldsymbol{q}) \boldsymbol{Z}^{T}(\boldsymbol{q})=\mathbf{0}$.

${ }^{4}$ I.e. it fulfills the condition $\boldsymbol{J}(\boldsymbol{q}) \boldsymbol{M}^{-1}(\boldsymbol{q}) \boldsymbol{N}^{T}(\boldsymbol{q})=\mathbf{0}$. 
where $v$ is an additional nullspace velocity. One can show that by this choice the extended Jacobian $\boldsymbol{J}_{N}(\boldsymbol{q})$ is non singular and

$$
\dot{\boldsymbol{q}}=\boldsymbol{J}_{N}^{-1}(\boldsymbol{q})\left[\begin{array}{l}
\dot{x} \\
v
\end{array}\right]=\left[\begin{array}{ll}
\boldsymbol{J}^{+M}(\boldsymbol{q}) & \boldsymbol{Z}^{T}(\boldsymbol{q})
\end{array}\right]\left[\begin{array}{c}
\dot{\boldsymbol{x}} \\
v
\end{array}\right],
$$

where $\boldsymbol{J}^{+M}(\boldsymbol{q})=\boldsymbol{M}^{-1}(\boldsymbol{q}) \boldsymbol{J}^{T}(\boldsymbol{q})\left(\boldsymbol{J}(\boldsymbol{q}) \boldsymbol{M}^{-1}(\boldsymbol{q}) \boldsymbol{J}^{T}(\boldsymbol{q})\right)^{-1}$ is the dynamically consistent weighted pseudo inverse.

From (6) and (7) it is straightforward to rewrite (3a) in the extended velocity coordinates as

$$
\boldsymbol{\Lambda}(\boldsymbol{q})\left[\begin{array}{c}
\ddot{x} \\
\dot{v}
\end{array}\right]+\boldsymbol{\Gamma}(\boldsymbol{q}, \dot{\boldsymbol{q}})\left[\begin{array}{l}
\dot{\boldsymbol{x}} \\
v
\end{array}\right]=\boldsymbol{J}_{N}^{-T}(\boldsymbol{q})[\boldsymbol{K}(\boldsymbol{\theta}-\boldsymbol{q})-\boldsymbol{g}(\boldsymbol{q})],
$$

with the matrices $\boldsymbol{\Lambda}(\boldsymbol{q})$ and $\boldsymbol{\Gamma}(\boldsymbol{q}, \dot{\boldsymbol{q}})$ given by ${ }^{5}$

$$
\begin{aligned}
& \boldsymbol{\Lambda}(\boldsymbol{q})=\left[\begin{array}{cc}
\boldsymbol{\Lambda}_{x}(\boldsymbol{q}) & \mathbf{0} \\
\mathbf{0} & \Lambda_{n}(\boldsymbol{q})
\end{array}\right] \\
& \boldsymbol{\Lambda}_{x}(\boldsymbol{q})=\left(\boldsymbol{J}(\boldsymbol{q}) \boldsymbol{M}^{-1}(\boldsymbol{q}) \boldsymbol{J}^{T}(\boldsymbol{q})\right)^{-1} \quad \Lambda_{n}(\boldsymbol{q})=\boldsymbol{Z}(\boldsymbol{q}) \boldsymbol{M}(\boldsymbol{q}) \boldsymbol{Z}^{T}(\boldsymbol{q})
\end{aligned}
$$

and (omitting the dependences)

$$
\begin{array}{ll}
\boldsymbol{\Gamma}(\boldsymbol{q}, \dot{\boldsymbol{q}})=\left[\begin{array}{cc}
\boldsymbol{\Gamma}_{x}(\boldsymbol{q}, \dot{\boldsymbol{q}}) & \boldsymbol{\Gamma}_{x n}(\boldsymbol{q}, \dot{\boldsymbol{q}}) \\
\boldsymbol{\Gamma}_{n x}(\boldsymbol{q}, \dot{\boldsymbol{q}}) & \Gamma_{n}(\boldsymbol{q}, \dot{\boldsymbol{q}})
\end{array}\right] & \\
\boldsymbol{\Gamma}_{x}=\boldsymbol{\Lambda}_{x}\left(\boldsymbol{J} \boldsymbol{M}^{-1} \boldsymbol{C}-\dot{\boldsymbol{J}}\right) \boldsymbol{J}^{+M} & \boldsymbol{\Gamma}_{x n}=\boldsymbol{\Lambda}_{x}\left(\boldsymbol{J} \boldsymbol{M}^{-1} \boldsymbol{C}-\dot{\boldsymbol{J}}\right) \mathbf{Z}^{T} \\
\boldsymbol{\Gamma}_{n x}=-\boldsymbol{\Gamma}_{x n}^{T} & \Gamma_{n}=\Lambda_{n}\left(\boldsymbol{N} \boldsymbol{M}^{-1} \boldsymbol{C}-\dot{\boldsymbol{N}}\right) \mathbf{Z}^{T} .
\end{array}
$$

Note that the change of coordinates does not alter the passivity property of the robot dynamics, i.e. $\dot{\Lambda}=\boldsymbol{\Gamma}+\boldsymbol{\Gamma}^{T}$ [19], [25], which will be used in the time differentiation of the Lyapunov functions introduced later on in the paper.

\section{Elastically actuated ROBOT}

In this section we present the main result of the paper. As in [6], [7], the limit cycle is generated regulating an energy function and forcing the system to evolve on a submanifold. Therefore, we will need to define an "energy-like" function, which in this case will take into account the physical potential energy stored in the springs. Compared to our previous works, the controller is itself a nonlinear dynamic system. Loosely speaking, the additional dynamics of the controller is used to regulate the energy of the system, while the motor dynamics is used to satisfy the virtual constraints. The latter is achieved through the input function $\tau_{d}$, introduced later, which has the meaning of a desired input to the link side equation. For this reason, it is similar to the control torque designed in [6], [7]. Before presenting the control law, it is necessary to extend some results to take into account that the system will be forced to evolve on a submanifold.

\section{A. The functions $\overline{\boldsymbol{q}}$ and $\overline{\boldsymbol{q}}_{x}$}

The linearity of the springs and (5) imply that a static mapping between motor and link positions exists, which provides for any motor position, the link position where the elastic elements in the joints compensate for the gravity term [12]. This link position is provided by the function $\overline{\boldsymbol{q}}$, defined

\footnotetext{
${ }^{5}$ Notice that using a dynamically consistent nullspace projector the matrix $\boldsymbol{\Lambda}(\boldsymbol{q})$ is block diagonal.
}

in [21], where it is shown that $\overline{\boldsymbol{q}}$ exists, is unique and a diffeomorphism.

The function $\overline{\boldsymbol{q}}$ can be generalized to take into account constraints on the allowed configurations of the robot. This is exactly what happens when the system is forced to evolve on a submanifold. To this end, we first provide an alternative definition of the function $\overline{\boldsymbol{q}}$ :

$$
\overline{\boldsymbol{q}}(\boldsymbol{\theta})=\arg \min _{\boldsymbol{q}} U(\boldsymbol{\theta}, \boldsymbol{q}) .
$$

Notice that the necessary condition for optimality of the minimization problem coincides with the definition of $\overline{\boldsymbol{q}}(\boldsymbol{\theta})$ in [21]. At this point the constraint is taken into account simply modifying the problem as

$$
\begin{array}{r}
\overline{\boldsymbol{q}}_{x}(\boldsymbol{\theta})=\arg \min _{\boldsymbol{q}} U(\boldsymbol{\theta}, \boldsymbol{q}) \\
\text { s.t. } \boldsymbol{x}(\boldsymbol{q})=\mathbf{0} .
\end{array}
$$

The necessary condition for optimality obtained using the Lagrange multipliers $\lambda$ is

$$
\nabla_{q} U(\boldsymbol{\theta}, \boldsymbol{q})+\boldsymbol{J}^{T}(\boldsymbol{q}) \lambda=0,
$$

so that pre-multiplying by the nullspace base matrix $\boldsymbol{Z}(\boldsymbol{q})$ we obtain $n(\boldsymbol{q})=0$, where $n(\boldsymbol{q})$ is defined as

$$
n(\boldsymbol{q}):=\boldsymbol{Z}(\boldsymbol{q}) \nabla_{\boldsymbol{q}} U(\boldsymbol{\theta}, \boldsymbol{q})=\boldsymbol{Z}(\boldsymbol{q})[\boldsymbol{g}(\boldsymbol{q})-\boldsymbol{K}(\boldsymbol{\theta}-\boldsymbol{q})]
$$

and can be seen as a local nullspace coordinate [26]. Concluding, $\overline{\boldsymbol{q}}_{x}(\boldsymbol{\theta})$ is the unique link configuration with coordinates $\boldsymbol{x}\left(\overline{\boldsymbol{q}}_{x}(\boldsymbol{\theta})\right)=\mathbf{0}, n\left(\overline{\boldsymbol{q}}_{x}(\boldsymbol{\theta})\right)=0$. This suggests that, from a computational point of view, the optimization problem can be solved setting up an inverse kinematic problem and finding $\overline{\boldsymbol{q}}_{x}(\boldsymbol{\theta})$ with a standard closed loop inverse kinematic scheme [25].

Proposition 2. The function $\overline{\boldsymbol{q}}_{x}(\boldsymbol{\theta})$, in a similar manner to $\overline{\boldsymbol{q}}(\boldsymbol{\theta})$, satisfies the properties

$$
\begin{array}{ll}
\text { - } U(\boldsymbol{\theta}, \boldsymbol{q}) \geq U\left(\boldsymbol{\theta}, \overline{\boldsymbol{q}}_{x}(\boldsymbol{\theta})\right) & \forall \boldsymbol{\theta}, \boldsymbol{q} \in \mathbb{Q}: \boldsymbol{x}(\boldsymbol{q})=\mathbf{0} \\
\text { - } U(\boldsymbol{\theta}, \boldsymbol{q})=U\left(\boldsymbol{\theta}, \overline{\boldsymbol{q}}_{x}(\boldsymbol{\theta})\right) \Leftrightarrow \boldsymbol{q}=\overline{\boldsymbol{q}}_{x}(\boldsymbol{\theta}) & \forall \boldsymbol{\theta}, \boldsymbol{q} \in \mathbb{Q}: \boldsymbol{x}(\boldsymbol{q})=\mathbf{0} \\
\text { - } \dot{U}\left(\boldsymbol{\theta}, \overline{\boldsymbol{q}}_{x}(\boldsymbol{\theta})\right)=\dot{\boldsymbol{\theta}}^{T} \boldsymbol{K}\left(\boldsymbol{\theta}-\overline{\boldsymbol{q}}_{x}(\boldsymbol{\theta})\right) &
\end{array}
$$

Proof. While the first two properties are a direct consequence of how the function is defined, for the third the following must be taken into account. By definition $\boldsymbol{x}\left(\overline{\boldsymbol{q}}_{x}(\boldsymbol{\theta})\right)=\mathbf{0}$ and therefore $\boldsymbol{J}\left(\overline{\boldsymbol{q}}_{x}(\boldsymbol{\theta})\right) \dot{\overline{\boldsymbol{q}}}_{x}(\boldsymbol{\theta})=\mathbf{0}, \forall \boldsymbol{\theta}(t) \in \mathbb{R}^{n}$. Using the chain rule it is possible to write:

$$
\dot{U}\left(\boldsymbol{\theta}, \overline{\boldsymbol{q}}_{x}(\boldsymbol{\theta})\right)=\left.\dot{\boldsymbol{\theta}}^{T} \nabla_{\boldsymbol{\theta}} U(\boldsymbol{\theta}, \boldsymbol{q})\right|_{\left(\boldsymbol{\theta}, \overline{\boldsymbol{q}}_{x}(\boldsymbol{\theta})\right)}+\left.\dot{\overline{\boldsymbol{q}}}_{x}^{T}(\boldsymbol{\theta}) \nabla_{\boldsymbol{q}} U(\boldsymbol{\theta}, \boldsymbol{q})\right|_{\left(\boldsymbol{\theta}, \overline{\bar{q}}_{x}(\boldsymbol{\theta})\right)} .
$$

The second term can be shown to be always zero since:

$$
\begin{aligned}
& \left.\dot{\overline{\boldsymbol{q}}}_{x}^{T}(\boldsymbol{\theta}) \boldsymbol{J}_{N}^{T}\left(\overline{\boldsymbol{q}}_{x}(\boldsymbol{\theta})\right) \boldsymbol{J}_{N}^{-T}\left(\overline{\boldsymbol{q}}_{x}(\boldsymbol{\theta})\right) \nabla_{\boldsymbol{q}} U(\boldsymbol{\theta}, \boldsymbol{q})\right|_{\left(\boldsymbol{\theta}, \overline{\boldsymbol{q}}_{x}(\boldsymbol{\theta})\right)}= \\
& {\left[\begin{array}{lll}
\mathbf{0} & \left.\dot{\boldsymbol{q}}_{x}^{T}(\boldsymbol{\theta}) \boldsymbol{N}^{T}\left(\overline{\boldsymbol{q}}_{x}(\boldsymbol{\theta})\right)\right]\left[\left.\nabla_{\boldsymbol{q}}^{T} U(\boldsymbol{\theta}, \boldsymbol{q})\right|_{\left(\boldsymbol{\theta}, \overline{\boldsymbol{q}}_{x}(\boldsymbol{\theta})\right)} \boldsymbol{J}^{+M}\left(\overline{\boldsymbol{q}}_{x}(\boldsymbol{\theta})\right)\right. & 0
\end{array}\right]^{T,},}
\end{aligned}
$$

where we have used $\boldsymbol{J}\left(\overline{\boldsymbol{q}}_{x}(\boldsymbol{\theta})\right) \dot{\overline{\boldsymbol{q}}}_{x}(\boldsymbol{\theta})=\mathbf{0}$ and $n\left(\overline{\boldsymbol{q}}_{x}(\boldsymbol{\theta})\right)=0$. The proof is concluded considering that only the potential energy due to the springs depends on $\boldsymbol{\theta}$ in the expression of the total potential $U(\boldsymbol{\theta}, \boldsymbol{q})$. 


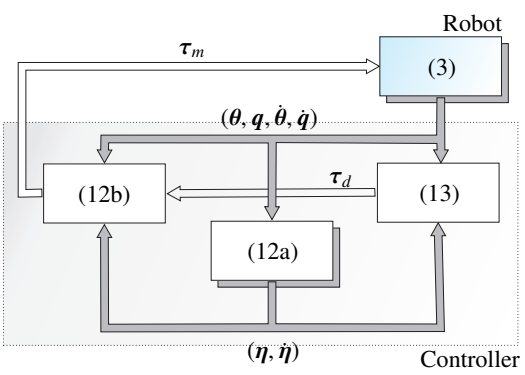

Fig. 2: Closed loop system.

\section{B. Main result}

Given the positive scalars $K_{H}, \alpha \in \mathbb{R}$ and the symmetric, positive definite matrices $\boldsymbol{K}_{\tau}, \boldsymbol{D}_{\tau}, \boldsymbol{K}_{\eta}, \boldsymbol{D}_{\eta} \in \mathbb{R}^{n \times n}$ and $\boldsymbol{K}_{x}, \boldsymbol{D}_{x} \in$ $\mathbb{R}^{(n-1) \times(n-1)}$, the proposed nonlinear dynamic state feedback controller is

$$
\begin{aligned}
\boldsymbol{B} \ddot{\boldsymbol{\eta}} & +K_{H} \tilde{H} \boldsymbol{K}\left(\overline{\boldsymbol{q}}_{x}(\boldsymbol{\eta})-\boldsymbol{q}\right)+\boldsymbol{D}_{\eta} \dot{\boldsymbol{\eta}}+\boldsymbol{K}_{\eta} \tilde{\boldsymbol{\eta}}=\mathbf{0} \\
\boldsymbol{\tau}_{m} & =\boldsymbol{K}(\boldsymbol{\theta}-\boldsymbol{q})-K_{H} \tilde{H} \boldsymbol{K}\left(\overline{\boldsymbol{q}}_{x}(\boldsymbol{\eta})-\boldsymbol{q}\right)-\boldsymbol{D}_{\eta} \dot{\boldsymbol{\eta}}-\boldsymbol{K}_{\eta} \tilde{\boldsymbol{\eta}} \\
& +\boldsymbol{B} \boldsymbol{K}^{-1}\left(\ddot{\boldsymbol{\tau}}_{d}-\boldsymbol{D}_{\tau} \dot{\tilde{\tau}}-\boldsymbol{K}_{\tau} \tilde{\boldsymbol{\tau}}\right)
\end{aligned}
$$

where $\boldsymbol{\eta}, \dot{\boldsymbol{\eta}} \in \mathbb{R}^{n}$ is the state, $\boldsymbol{\tau}_{m} \in \mathbb{R}^{n}$ is the controller output function and $\tilde{\boldsymbol{\tau}}=\boldsymbol{K}(\boldsymbol{\theta}-\boldsymbol{\eta})-\boldsymbol{\tau}_{d}$ being $\boldsymbol{\tau}_{d} \in \mathbb{R}^{n}$ the controller input function. The latter is computed based on the state of the system and of the controller itself and it is given by

$$
\begin{aligned}
\boldsymbol{\tau}_{d} & =\boldsymbol{J}_{N}^{T}(\boldsymbol{q})\left[\begin{array}{cc}
\boldsymbol{J}^{+{ }_{M} T}(\boldsymbol{q})[\boldsymbol{g}(\boldsymbol{q})-\boldsymbol{K}(\boldsymbol{\eta}-\boldsymbol{q})]-\boldsymbol{K}_{x} \boldsymbol{x}(\boldsymbol{q}) \\
\mathbf{0}
\end{array}\right] \\
& +\boldsymbol{J}_{N}^{T}(\boldsymbol{q})\left[\begin{array}{cc}
-\boldsymbol{D}_{x} & \boldsymbol{\Gamma}_{x n}(\boldsymbol{q}, \dot{\boldsymbol{q}})-\boldsymbol{\alpha} \dot{\boldsymbol{x}} v \\
-\boldsymbol{\Gamma}_{x n}^{T}(\boldsymbol{q}, \dot{\boldsymbol{q}})+\boldsymbol{\alpha} v \dot{\boldsymbol{x}}^{T} & \boldsymbol{O}
\end{array}\right]\left[\begin{array}{c}
\dot{\boldsymbol{x}} \\
v
\end{array}\right],
\end{aligned},
$$

where $\tilde{\boldsymbol{\eta}}=\boldsymbol{\eta}-\boldsymbol{\eta}_{d}, \boldsymbol{\eta}_{d} \in \mathbb{R}^{n}$ is a constant and

$$
H(\chi):=\frac{1}{2} \dot{\boldsymbol{q}}^{T} \boldsymbol{M} \dot{\boldsymbol{q}}+U(\boldsymbol{\eta}, \boldsymbol{q})-U\left(\boldsymbol{\eta}, \overline{\boldsymbol{q}}_{x}(\boldsymbol{\eta})\right) .
$$

Interconnecting the system (3) with the controller (12) as in Fig. 2, we obtain the closed loop system:

$$
\begin{aligned}
& \boldsymbol{M}(\boldsymbol{q}) \ddot{\boldsymbol{q}}+\boldsymbol{C}(\boldsymbol{q}, \dot{\boldsymbol{q}}) \dot{\boldsymbol{q}}+\boldsymbol{g}(\boldsymbol{q})=\boldsymbol{K}(\boldsymbol{\eta}-\boldsymbol{q})+\boldsymbol{\tau}_{d}+\tilde{\boldsymbol{\tau}} \\
& \ddot{\tilde{\tau}}+\boldsymbol{D}_{\tau} \dot{\tilde{\tau}}+\boldsymbol{K}_{\tau} \tilde{\boldsymbol{\tau}}=\mathbf{0} \\
& \boldsymbol{B} \ddot{\boldsymbol{\eta}}+K_{H} \tilde{H} \boldsymbol{K}\left(\overline{\boldsymbol{q}}_{x}(\boldsymbol{\eta})-\boldsymbol{q}\right)+\boldsymbol{D}_{\eta} \dot{\boldsymbol{\eta}}+\boldsymbol{K}_{\eta} \tilde{\boldsymbol{\eta}}=\mathbf{0},
\end{aligned}
$$

where $(12 b)$ has been replaced in (3b). Using the state vector $\chi=(\boldsymbol{q}, \dot{\boldsymbol{x}}, v, \tilde{\tau}, \dot{\tilde{\tau}}, \boldsymbol{\eta}, \dot{\boldsymbol{\eta}})$, the input function (13) and the coordinate transformation in Section III, the closed loop system can be rewritten as (omitting the dependences):

$$
\begin{aligned}
& \dot{\boldsymbol{q}}=\boldsymbol{J}^{{ }{ }^{M}} \dot{\boldsymbol{x}}+\boldsymbol{Z}^{T} v \\
& \boldsymbol{\Lambda}_{x} \ddot{\boldsymbol{x}}+\left(\boldsymbol{\Gamma}_{x}+\boldsymbol{D}_{x}+\boldsymbol{\alpha} v^{2} \boldsymbol{I}\right) \dot{\boldsymbol{x}}+\boldsymbol{K}_{x} \boldsymbol{x}=\boldsymbol{J}^{+{ } T} \tilde{\boldsymbol{\tau}} \\
& \Lambda_{n} \dot{v}+\left(\Gamma_{n}-\boldsymbol{\alpha} \dot{\boldsymbol{x}}^{T} \dot{\boldsymbol{x}}\right) v+\boldsymbol{Z} \boldsymbol{g}=\boldsymbol{Z}[\boldsymbol{K}(\boldsymbol{\eta}-\boldsymbol{q})+\tilde{\boldsymbol{\tau}}] \\
& \ddot{\tilde{\boldsymbol{\tau}}}+\boldsymbol{D}_{\tau} \dot{\tilde{\tau}}+\boldsymbol{K}_{\tau} \tilde{\boldsymbol{\tau}}=\mathbf{0} \\
& \boldsymbol{B} \ddot{\boldsymbol{\eta}}+K_{H} \tilde{H} \boldsymbol{K}\left(\overline{\boldsymbol{q}}_{x}(\boldsymbol{\eta})-\boldsymbol{q}\right)+\boldsymbol{D}_{\eta} \dot{\boldsymbol{\eta}}+\boldsymbol{K}_{\eta} \tilde{\boldsymbol{\eta}}=\mathbf{0} .
\end{aligned}
$$

The stability properties of the system are summarized in the following theorem.

Theorem 1. Given (5), the nonlinear autonomous system (16) has an asymptotically stable solution consisting of

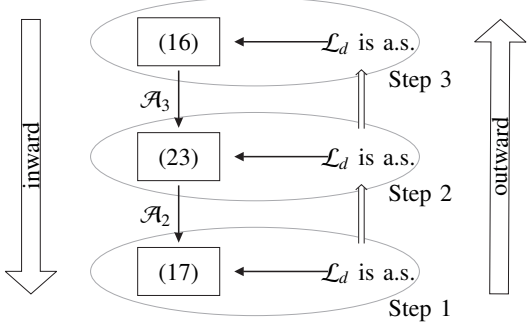

Fig. 3: Flow of the argumentation used in the proof.

(a) the equilibrium point $\chi^{*}=\left(\overline{\boldsymbol{q}}_{x}\left(\boldsymbol{\eta}_{d}\right), \mathbf{0}, 0, \mathbf{0}, \mathbf{0}, \boldsymbol{\eta}_{d}, \mathbf{0}\right)$ for $H_{d}=0$

(b) the limit cycle defined by

$$
\mathcal{L}_{d}=\{\chi \mid \tilde{H}(\chi)=0, \boldsymbol{x}(\boldsymbol{q})=\dot{\boldsymbol{x}}=\mathbf{0}, \tilde{\boldsymbol{\eta}}=\dot{\boldsymbol{\eta}}=\tilde{\boldsymbol{\tau}}=\dot{\tilde{\tau}}=\mathbf{0}\}
$$

for $H_{d}>0$.

Proof. The proof is split in three parts, since we will use twice Theorem 3 reported in the Appendix. We will use a semidefinite Lyapunov function $V_{3}(\chi)$ to carry on the stability analysis of (16). Therefore, we consider the system conditionally to the largest invariant set within the set where $\dot{V}_{3}=0$, i.e. (23). Once again, we will use a semidefinite Lyapunov function $V_{2}$ to carry on the stability analysis of (23). Therefore, we consider the system conditionally to the largest invariant set within the set where $\dot{V}_{2}=0$, i.e. (17). We will start considering the most inner system and then go outwards till (16) after two steps. The flow of the argumentation used in the proof is shown in Fig. 3.

1) Step 1: We first show that the nonlinear autonomous system

$$
\begin{aligned}
& \dot{\boldsymbol{q}}=\boldsymbol{Z}^{T} v \\
& \Lambda_{n} \dot{v}+\Gamma_{n} v+\boldsymbol{Z} \boldsymbol{g}=\boldsymbol{Z} \boldsymbol{K}(\boldsymbol{\eta}-\boldsymbol{q}) \\
& \boldsymbol{B} \ddot{\boldsymbol{\eta}}+K_{H} \tilde{H} \boldsymbol{K}\left(\overline{\boldsymbol{q}}_{x}(\boldsymbol{\eta})-\boldsymbol{q}\right)+\boldsymbol{D}_{\eta} \dot{\boldsymbol{\eta}}+\boldsymbol{K}_{\eta} \tilde{\boldsymbol{\eta}}=\mathbf{0}
\end{aligned}
$$

with $\chi \in \mathcal{A}_{2}=\{\chi \mid \tilde{\boldsymbol{\tau}}=\dot{\tilde{\tau}}=\mathbf{0}, \boldsymbol{x}(\boldsymbol{q})=\dot{\boldsymbol{x}}=\mathbf{0}\}$, will always reach the set $\mathcal{L}_{d}$ and therefore

- has an asymptotically stable equilibrium point in $\chi^{*}$ for $H_{d}=0$

- has an asymptotically stable limit cycle defined by $\mathcal{L}_{d}$ for $H_{d}>0$.

This is proven showing invariance, stability and finally attractiveness of $\mathcal{L}_{d}$.

Invariance: Given the properties of $\overline{\boldsymbol{q}}_{x}, H(\chi)$ is an "energy-like" function for (17). Additionally, computing the time derivative of $\tilde{H}$ along the flow of (17), results in

$$
\dot{\tilde{H}}(\chi)=\dot{H}(\chi)=\dot{\boldsymbol{\eta}}^{T}\left[\boldsymbol{K}(\boldsymbol{\eta}-\boldsymbol{q})-\boldsymbol{K}\left(\boldsymbol{\eta}-\overline{\boldsymbol{q}}_{x}(\boldsymbol{\eta})\right)\right] .
$$

Since $\dot{\boldsymbol{\eta}}=\mathbf{0} \Longrightarrow \dot{\tilde{H}}=0$, we conclude that $\mathcal{L}_{d}$ is an invariant set for (17), because, starting from $\mathcal{L}_{d}, \tilde{H}$ will not change.

Stability: The $C^{1}$ function of the state

$$
V_{1}(\chi)=\frac{1}{2}\left(K_{H} \tilde{H}^{2}(\chi)+\dot{\boldsymbol{\eta}}^{T} \boldsymbol{B} \dot{\boldsymbol{\eta}}+\tilde{\boldsymbol{\eta}}^{T} \boldsymbol{K}_{\eta} \tilde{\boldsymbol{\eta}}\right)
$$


is such that

$$
\begin{cases}V_{1}(\chi)=0 & \forall \chi \in \mathcal{L}_{d} \\ V_{1}(\chi)>0 & \forall \chi \notin \mathcal{L}_{d}\end{cases}
$$

and therefore it is a candidate Lyapunov function to prove the stability of $\mathcal{L}_{d}$ for (17). Computing its derivative along the flow of the system (17), leads to $\dot{V}_{1}(\chi)=-\dot{\boldsymbol{\eta}}^{T} \boldsymbol{D}_{\eta} \dot{\boldsymbol{\eta}} \leq 0$, which ensures the stability of $\mathcal{L}_{d}$ for the system (17).

Using LaSalle's invariance principle we can conclude that the positive limit set for the solutions of (17) is given by $M_{1}=\left\{\chi^{*}, \mathcal{L}_{d}\right\}$, because $M_{1}$ is the largest positive invariant set within the set $E_{1}=\{\chi \mid \dot{\boldsymbol{\eta}}=\mathbf{0}\}$, i.e. the set where $\dot{V}_{1}(\chi)=0$. This will be shown in two subsequent steps, starting from the condition $\dot{\boldsymbol{\eta}}=\mathbf{0}$.

Invariant set: First we show that $\dot{\boldsymbol{\eta}}=\mathbf{0} \Rightarrow \tilde{\boldsymbol{\eta}}=\mathbf{0}$. From $\dot{\boldsymbol{\eta}}=\mathbf{0}$ we can directly conclude that $\boldsymbol{\eta}=\boldsymbol{\eta}_{0}$ and, because of (18), also $H(\chi)=H_{0}$, where $\boldsymbol{\eta}_{0}$ and $H_{0}$ are constants. Moreover, from (17c) we get

$$
-K_{H}\left(H_{0}-H_{d}\right) \boldsymbol{K}\left(\overline{\boldsymbol{q}}_{x}\left(\boldsymbol{\eta}_{0}\right)-\boldsymbol{q}\right)-\boldsymbol{K}_{\eta}\left(\boldsymbol{\eta}_{0}-\boldsymbol{\eta}_{d}\right)=\mathbf{0},
$$

from which two cases are possible

$$
\left\{\begin{array}{l}
H_{0}=H_{d} \\
H_{0} \neq H_{d}
\end{array}\right.
$$

While from the first we can directly conclude $\boldsymbol{\eta}_{0}=\boldsymbol{\eta}_{d}$, in the second case we conclude that $\boldsymbol{q}=\boldsymbol{q}_{0}$, where $\boldsymbol{q}_{0}$ is constant, since all the quantities in (20) are constants. This implies that $v=0$ and therefore from (17b) we get

$$
\boldsymbol{Z}\left(\boldsymbol{q}_{0}\right)\left[\boldsymbol{g}\left(\boldsymbol{q}_{0}\right)-\boldsymbol{K}\left(\boldsymbol{\eta}_{0}-\boldsymbol{q}_{0}\right)\right]=0 .
$$

In addition, we know that $\boldsymbol{x}\left(\boldsymbol{q}_{0}\right)=\mathbf{0}$, or in other words $\boldsymbol{q}_{0}=$ $\overline{\boldsymbol{q}}_{x}\left(\boldsymbol{\eta}_{0}\right)$ and therefore from (20) also in this case $\boldsymbol{\eta}_{0}=\boldsymbol{\eta}_{d}$.

Now we finally prove that $\dot{\boldsymbol{\eta}}=\tilde{\boldsymbol{\eta}}=\mathbf{0} \Rightarrow M_{1}=\left\{\chi^{*}, \mathcal{L}_{d}\right\}$. In addition to the previous conditions we have $\boldsymbol{\eta}=\boldsymbol{\eta}_{d}$, therefore from $(17 \mathrm{c})$ it follows that

$$
-K_{H}\left(H_{0}-H_{d}\right) \boldsymbol{K}\left(\overline{\boldsymbol{q}}_{x}\left(\boldsymbol{\eta}_{d}\right)-\boldsymbol{q}\right)=\mathbf{0} .
$$

If $H_{0}=H_{d}$ we get $\mathcal{L}_{d}$ by definition, while in case $H_{0} \neq H_{d}$ then $\boldsymbol{q}=\overline{\boldsymbol{q}}_{x}\left(\boldsymbol{\eta}_{d}\right)$ and consequently $\dot{\boldsymbol{x}}=\mathbf{0}, v=0$ or in other words $\chi=\chi^{*}$.

Asymptotic stability: Since $\mathcal{L}_{d}$ is stable and attractive, we conclude that $\mathcal{L}_{d}$ is asymptotically stable. Additionally, since

$$
H(\chi)=0 \Longleftrightarrow \boldsymbol{q}=\overline{\boldsymbol{q}}_{x}(\boldsymbol{\eta}), v=0
$$

$\forall \chi \in \mathcal{A}_{2}$, it can be easily verified that $\mathcal{L}_{d}$ coincides with $\chi^{*}$ when $H_{d}=0$, which becomes the only positive limit set and therefore result $(a)$ is obtained. On the other hand, when $H_{d}>0$, the set $\mathcal{L}_{d}$ is uniquely determined by one parameter,

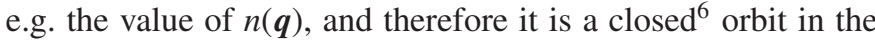
state space, i.e. a limit cycle, proving result $(b)$.

Given this result, in the reminder of the proof we will simply refer to the stability property of the set $\mathcal{L}_{d}$, with the conditions (a) and (b) arising naturally depending on the value of $H_{d}$.

${ }^{6}$ It is the level set of a positive definite and radially unbounded function.
2) Step 2: Given the nonlinear autonomous system

$$
\begin{aligned}
& \dot{\boldsymbol{q}}=\boldsymbol{J}^{+M} \dot{\boldsymbol{x}}+\boldsymbol{Z}^{T} v \\
& \boldsymbol{\Lambda}_{x} \ddot{\boldsymbol{x}}+\left(\boldsymbol{\Gamma}_{x}+\boldsymbol{D}_{x}+\boldsymbol{\alpha} v^{2} \boldsymbol{I}\right) \dot{\boldsymbol{x}}+\boldsymbol{K}_{x} \boldsymbol{x}=\mathbf{0} \\
& \Lambda_{n} \dot{v}+\left(\Gamma_{n}-\boldsymbol{\alpha} \dot{\boldsymbol{x}}^{T} \dot{\boldsymbol{x}}\right) v+\boldsymbol{Z} \boldsymbol{g}=\boldsymbol{Z} \boldsymbol{K}(\boldsymbol{\eta}-\boldsymbol{q}) \\
& \boldsymbol{B} \ddot{\boldsymbol{\eta}}+K_{H} \tilde{H} \boldsymbol{K}\left(\overline{\boldsymbol{q}}_{x}(\boldsymbol{\eta})-\boldsymbol{q}\right)+\boldsymbol{D}_{\eta} \dot{\boldsymbol{\eta}}+\boldsymbol{K}_{\eta} \tilde{\boldsymbol{\eta}}=\mathbf{0},
\end{aligned}
$$

with $\chi \in \mathcal{A}_{3}=\{\chi \mid \tilde{\tau}=\dot{\tilde{\tau}}=\mathbf{0}\}$, let us consider the $C^{1}$ function of the state

$$
V_{2}(\chi)=\frac{1}{2}\left(\dot{\boldsymbol{x}}^{T} \boldsymbol{\Lambda}_{x}(\boldsymbol{q}) \dot{\boldsymbol{x}}+\boldsymbol{x}^{T}(\boldsymbol{q}) \boldsymbol{K}_{x} \boldsymbol{x}(\boldsymbol{q})\right),
$$

such that

$$
\begin{cases}V_{2}(\chi)=0 & \forall \chi \in \mathcal{A}_{2} \\ V_{2}(\chi)>0 & \forall \chi \notin \mathcal{A}_{2}\end{cases}
$$

Since $\mathcal{L}_{d} \subset \mathcal{A}_{2}, V_{2}(\chi)$ is a candidate semidefinite Lyapunov function to prove the stability of $\mathcal{L}_{d}$ for (23). Computing its derivative along the flow of the system, leads to $\dot{V}_{2}(\chi)=$ $-\dot{\boldsymbol{x}}^{T}\left(\boldsymbol{D}_{x}+\boldsymbol{\alpha} v^{2} \boldsymbol{I}\right) \dot{\boldsymbol{x}} \leq 0$. The set $\mathcal{A}_{2}$ is the largest positively invariant set within $E_{2}=\{\chi \mid \dot{\boldsymbol{x}}=\mathbf{0}\}$, i.e. the set where $\dot{V}_{2}(\chi)=0$. In fact, if $\boldsymbol{x}(\boldsymbol{q}) \neq \mathbf{0}$ then we leave $E_{2}$ as it can be seen from (23b). Finally, since $\mathcal{L}_{d}$ is asymptotically stable conditionally to $\mathcal{A}_{2}$ (which is exactly what we have proven in Paragraph IV-B1), then by Theorem $3 \mathcal{L}_{d}$ is asymptotically stable for (23).

3) Step 3: Let us consider for the system (16) the $C^{1}$ function of the state

$$
V_{3}(\chi)=\frac{1}{2}\left(\dot{\tilde{\tau}}^{T} \dot{\tilde{\tau}}+\tilde{\boldsymbol{\tau}}^{T} \boldsymbol{K}_{\tau} \tilde{\tau}\right)
$$

such that

$$
\begin{cases}V_{3}(\chi)=0 & \forall \chi \in \mathcal{A}_{3} \\ V_{3}(\chi)>0 & \forall \chi \notin \mathcal{A}_{3} .\end{cases}
$$

Since $\mathcal{L}_{d} \subset \mathcal{A}_{3}, V_{3}(\chi)$ is a candidate semidefinite Lyapunov function to prove the stability of $\mathcal{L}_{d}$ for (16). Computing its derivative along the flow of the system, leads to $\dot{V}_{3}(\chi)=$ $-\dot{\tilde{\tau}}^{T} \boldsymbol{D}_{\tau} \dot{\tilde{\tau}} \leq 0$. The set $\mathcal{A}_{3}$ is the largest positively invariant set within $E_{3}=\{\chi \mid \dot{\tilde{\tau}}=\mathbf{0}\}$, i.e. the set where $\dot{V}_{3}(\chi)=0$. In fact, if $\tilde{\boldsymbol{\tau}} \neq \mathbf{0}$ then we leave $E_{3}$ as it can be seen from (16d). Finally, since $\mathcal{L}_{d}$ is asymptotically stable conditionally to $\mathcal{A}_{3}$ (which is exactly what we have proven in Paragraph IV-B2), then by Theorem $3 \mathcal{L}_{d}$ is asymptotically stable for (16).

\section{Controller discussion}

It is possible to recognize different contributions in $\tau_{d}$ : the torque responsible for forcing the system to evolve on the constraint submanifold, the one compensating for the coupling terms in the Coriolis matrix and the one shifting energy from the constraint space to the nullspace (i.e. the terms depending on $\alpha$ ). Only the part of the gravitational torque which causes the system to go off the submanifold are compensated compared to [6], [7], since the gravitational potential energy itself is used to produce the oscillation. Additionally, the limit cycle is produced involving directly the springs in the joints. The torque that they produce is split in the one necessary to keep the system to evolve on the submanifold and the one that 


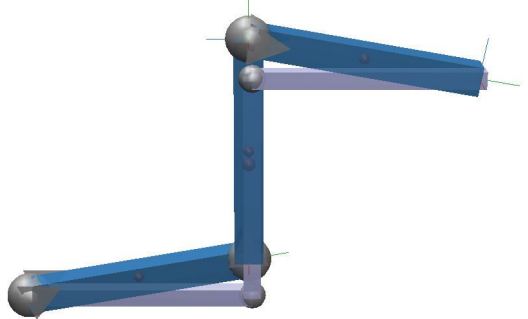

Fig. 4: Starting configuration of the robot ed equilibrium configuration (lighter color).

can be used to produce the limit cycle. This is achieved via the control state, which by mimicking the motor dynamics, is acting as a rest length adjustment.

The output function (12b) of the controller requires up to the second derivative of the input function $\tau_{d}$. Since the latter is a function of $\boldsymbol{q}, \dot{\boldsymbol{q}}$ and $\boldsymbol{\eta}$, in addition to the state, it is required the knowledge of the link acceleration $\ddot{\boldsymbol{q}}$, the jerk $\boldsymbol{q}^{(3)}$ and $\ddot{\boldsymbol{\eta}}$. While the signal $\ddot{\boldsymbol{\eta}}$ is easily computed, i.e. through (16e), since the model of the controller can be reasonably assumed to be known, the same is not true for the link acceleration and jerk. One can compute these signals based on the model equations or alternatively, directly through acceleration sensors and appropriate filtering techniques. From an implementation prospective, this is a weak point, which is although shared by all the control laws that use the motors to make the torque produced by the springs track a desired one (i.e. using the rigid case as an intermediate design step [19, Chapter 6]). In our approach, this aspect is less problematic since the torque $\tau_{d}$ that needs to be tracked is responsible only to guarantee the convergence to the invariant set of the state space and not for the regulation of the energy, as it will be clear with the results shown in Section V.

Although the proposed controller is model based, an analysis in case of uncertainties is beyond the scope of this work. Nevertheless, preliminary results on adaptive friction compensation can be found in [7].

\section{Simulations}

In this section we report some simulation results, to validate the proposed control approach. The simulation implements the model in (3) and uses the algorithm and formulas from [27] both to compute the control law and for the forward dynamics. No additional control input constraints are included.

As case study we will consider a 3 - link robotic arm, with each link having the properties reported in Table I. The robot starts from the configuration shown in Fig 4.

TABLE I: Properties of one of the three modules of the robot.

\begin{tabular}{lll|ll} 
Link & & & Spring & Motor \\
\hline Length & Mass & Inertia & Stiffness & Inertia \\
\hline $0.4 \mathrm{~m}$ & $5 \mathrm{~kg}$ & $0.2 \mathrm{kgm}^{2}$ & $200 \mathrm{Nm} / \mathrm{rad}$ & $0.6325 \mathrm{kgm}^{2}$
\end{tabular}

The function $\boldsymbol{x}(\boldsymbol{q})$ is chosen as

$$
\begin{aligned}
& x_{1}(\boldsymbol{q})=z(\boldsymbol{q})-0.8 \\
& x_{2}(\boldsymbol{q})=\psi(\boldsymbol{q}),
\end{aligned}
$$

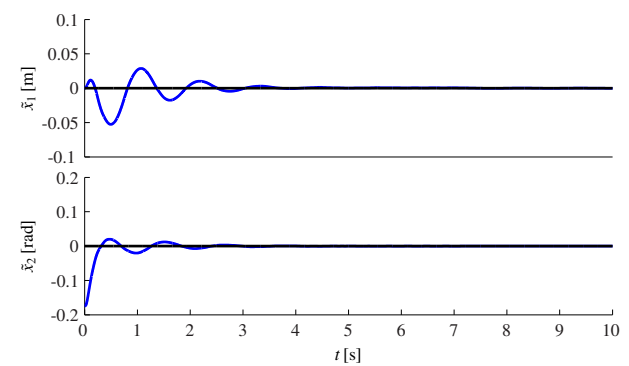

Fig. 5: Convergence to zero of the two components of the constraint function $\boldsymbol{x}(\boldsymbol{q})$.

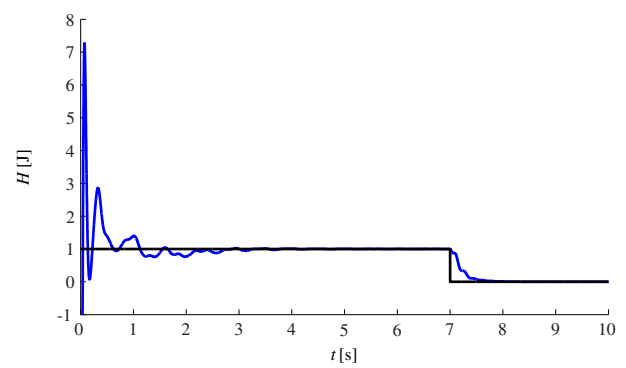

Fig. 6: Energy function (14) (solid line) and desired value (dashed line).

where $z(\boldsymbol{q})$ is the vertical position and $\psi(\boldsymbol{q})$ the orientation of the end - effector which, given the definition of $\boldsymbol{x}(\boldsymbol{q})$, is required to stay at a height of $0.8 \mathrm{~m}$ from the floor and to keep the orientation parallel to it. The convergence of $\boldsymbol{x}(\boldsymbol{q})$ to zero is shown in Fig. 5.

The desired value of the energy is switched from $H_{d}=1 \mathrm{~J}$ to zero in order to show how the oscillation can be produced and then damped out. Given the definition of $\boldsymbol{x}(\boldsymbol{q})$, the resulting motion will be an horizontal oscillation when $H_{d}>0$ while the robot will stop at the equilibrium when $H_{d}=0$. The evolution of the energy function is shown in Fig. 6. As it can be seen, the energy is effectively regulated to the desired value, whose sudden variation has no influence on $\boldsymbol{x}(\boldsymbol{q})$.

In order to highlight the role of $\alpha$ appearing in $\tau_{d}$, in Fig. 7 and Fig. 8 we show the total kinetic energy and its two contributions (i.e. the kinetic energy in the constraint space and nullspace) for $\alpha=0$ and $\alpha=30$ respectively. As it can be noticed, in the second case the kinetic energy in the constraint space converges more rapidly to zero, while the one in the nullspace increases faster.

Table II collects the values of all the gains used in the simulation.

TABLE II: Gains used in the simulation. When only the $i$ - th

\begin{tabular}{|c|c|c|c|c|}
\hline$K_{x_{1}}$ & $D_{x_{1}}$ & $K_{x_{2}}$ & $D_{x_{2}}$ & \\
\hline $400 \mathrm{~N} / \mathrm{m}$ & $36 \mathrm{~N} / \mathrm{ms}$ & $400 \mathrm{Nm} / \mathrm{rad}$ & $36 \mathrm{Nms} / \mathrm{rad}$ & \\
\hline$K_{\tau_{i}}$ & $D_{\tau_{i}}$ & $K_{\eta_{i}}$ & $D_{\eta_{i}}$ & $K_{H}$ \\
\hline $16001 / \mathrm{s}^{2}$ & $641 / \mathrm{s}$ & $100 \mathrm{Nm} / \mathrm{rad}$ & $16 \mathrm{Nms} / \mathrm{rad}$ & $0.3 \mathrm{~s}^{2} / \mathrm{kgm}^{2}$ \\
\hline
\end{tabular}
entry is shown, then the others have the same value.

Before concluding the section, the control law proposed in Section IV is compared to a cascaded design [19, Chapter 6], in which the desired torque is chosen as in [6], with a 


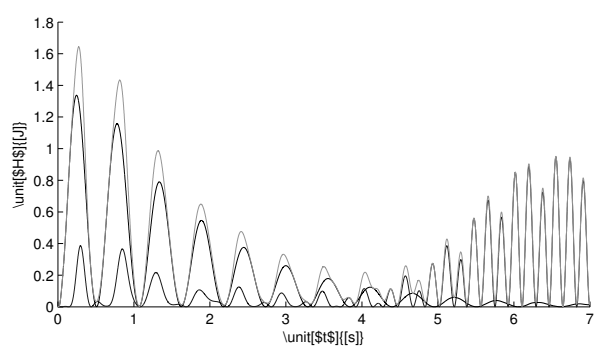

Fig. 7: Total kinetic energy (black line) and its two components, i.e. kinetic energy in the constraint space (dark-grey line) an in the nullspace (light-grey line), in case $\alpha=0$.

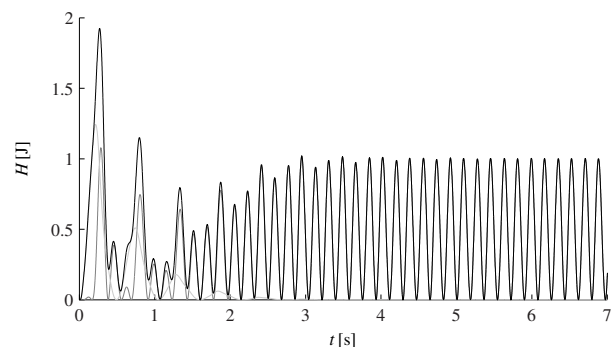

Fig. 8: Total kinetic energy (black line) and its two components, i.e. kinetic energy in the constraint space (dark-grey line) an in the nullspace (light-grey line), in case $\alpha=30$.

virtual potential given by $U\left(\boldsymbol{\eta}_{d}, \boldsymbol{q}\right)-U\left(\boldsymbol{\eta}_{d}, \overline{\boldsymbol{q}}_{x}\left(\boldsymbol{\eta}_{d}\right)\right)$. In this case similar performances can be achieved at the price of using considerably larger gains $\boldsymbol{K}_{\tau}$ and $\boldsymbol{D}_{\tau}$. Obviously, this can compromise the implementation on a real system. The results shown in Fig. 9 can be interpreted as follows: in the control law proposed in Section IV, it is not necessary to track a desired torque to generate the limit cycle, but only to force the system to evolve on a submanifold. The latter is not varying as rapidly as the one necessary to produce the limit cycle, therefore smaller gains are sufficient. Additionally, the tracking of the desired torque in the cascaded design has a more direct effect on the regulation of the energy, since an imperfect tracking will directly result in the incapability for the system to reach the desired value of the energy.

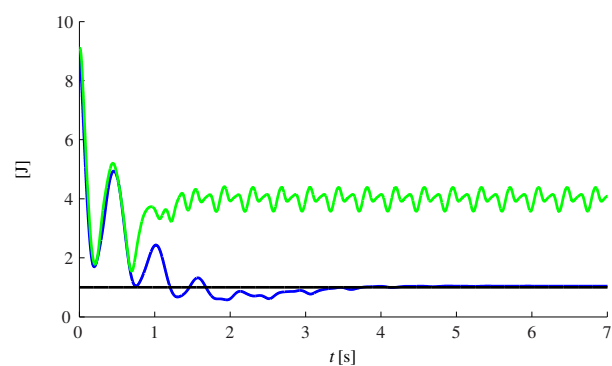

Fig. 9: Energy function in [6] for $K_{\tau_{i}}=10^{4} 1 / \mathrm{s}^{2}, D_{\tau_{i}}=1601 / \mathrm{s}$ (light-grey line), $K_{\tau_{i}}=10^{8} 1 / \mathrm{s}^{2}, D_{\tau_{i}}=16^{3} 1 / \mathrm{s}$ (dark-grey line) and desired value (black line).

\section{Conclusion}

In this paper we have presented a new control law for elastic joint robots capable of utilizing the energy stored by the springs in order to produce efficiently an asymptotically stable limit cycle for the closed loop system. At the same time, by simply setting the desired value of the energy to zero, the same control law can be used to regulate the robot to a desired configuration. Since elastic joint robots are underactuated mechanical systems, there are not enough control inputs to easily force the system to evolve on the submanifold and regulate at the same time the energy to the desired value. Loosely speaking, the issue is solved by introducing additional dynamics through the controller. As a result, at the end of our design process we obtain a nonlinear dynamic state feedback controller. Finally, for the stability analysis, we make extensively use of the semidefinite Lyapunov theory in order to reduce the system in subsystems, which are easier to analyze.

As part of the future work, we plan to apply the proposed control approach on a compliant bipedal robot, with linear springs in each joint, which is currently under development. The control law proposed in this paper can be used as a periodic motion generator, which needs to be combined with balancing and foot placement strategies to achieve hopping and running.

\section{APPENDIX}

The results presented here are based on [6], [28], [29].

Given $\chi \in \mathcal{X} \subset \mathbb{R}^{m}$ and a Lipschitz continuous function $f: \mathcal{X} \rightarrow \mathbb{R}^{m}$, the system

$$
\dot{\chi}=f(\chi)
$$

has a unique solution starting at $\chi_{0}$ and evaluated at the time instant $t$ that we denote with $\chi\left(t ; \chi_{0}\right)$. Additionally, given an invariant set $\Omega$ for (26), we define:

a) (Distance): $d(\chi, \Omega) \triangleq \min _{y \in \Omega}\|\chi-y\|$

b) (Open ball): $B_{\epsilon}(\Omega) \triangleq\{\chi \in \mathcal{X} \mid d(\chi, \Omega)<\epsilon\}$

c) (Sphere): $S_{\epsilon}(\Omega) \triangleq\{\chi \in \mathcal{X} \mid d(\chi, \Omega)=\epsilon\}$

Theorem 2 (Stability). Let $\Omega$ be a bounded, invariant set for (26), and let $V(\chi)$ be a $C^{1}$ function such that $V(\chi) \geq 0, V(\Omega)=$ 0 and $\dot{V}(\chi) \leq 0$. If $\Omega$ is asymptotically stable conditionally to $\mathcal{A}=\{\chi \mid V(\chi)=0\}$, then $\Omega$ is stable.

Proof. Suppose by contradiction that $\Omega$ is unstable. Then exist an $\epsilon$ such that $v>\epsilon>0$, a sequence $\left(\chi_{0 n}\right)_{n \in \mathbb{N}} \subset B_{\epsilon}(\Omega)$, $\lim _{n \rightarrow \infty} d\left(\chi_{0 n}, \Omega\right)=0$, and a sequence $\left(t_{n}\right)_{n \in \mathbb{N}} \subset \mathbb{R}^{+}$in such a way that

$$
\begin{cases}d\left(\chi\left(t ; \chi_{0 n}\right), \Omega\right)<\epsilon & 0 \leq t<t_{n} \\ d\left(\chi\left(t_{n} ; \chi_{0 n}\right), \Omega\right)=\epsilon & \forall n \in \mathbb{N}\end{cases}
$$

Since $S_{\epsilon}(\Omega)$ is compact, we can extract a convergent subsequence $\boldsymbol{y}_{n}^{\prime}$ from $\boldsymbol{y}_{n}=\chi\left(t_{n} ; \chi_{0 n}\right)$ such that $\boldsymbol{y}_{n}^{\prime} \rightarrow \boldsymbol{y} \in S_{\epsilon}(\Omega)$ as $n \rightarrow \infty$. Moreover because of the continuity of the solutions of (26) and the invariance of $\Omega, t_{n} \rightarrow \infty$ as $n \rightarrow \infty$.

Now we show that $V(\chi(-t ; \boldsymbol{y}))=0$. Let $\tau<0$ and $N \in \mathbb{N}$ be such that $0<t_{n}+\tau<t_{n}, \forall n \geq N$. Because $V$ is not increasing along the solutions of (26), we have that

$$
0 \leq V\left(\chi\left(t_{n}+\tau ; \chi_{0 n}\right)\right) \leq V\left(\chi_{0 n}\right) .
$$


From $\lim _{n \rightarrow \infty} d\left(\chi_{0 n}, \Omega\right)=0, V(\Omega)=0$ and the continuity of $V$, it follows

$$
\begin{aligned}
V(\chi(\tau ; \boldsymbol{y})) & =\lim _{n \rightarrow \infty} V\left(\chi\left(\tau ; \chi\left(t_{n} ; \chi_{0 n}\right)\right)\right) \\
& =\lim _{n \rightarrow \infty} V\left(\chi\left(t_{n}+\tau ; \chi_{0 n}\right)\right)=0 .
\end{aligned}
$$

It remains to prove that $\chi(-t ; \boldsymbol{y}) \in \mathcal{A}$ and $d(\boldsymbol{y}, \Omega)=\epsilon$ cannot hold if $\Omega$ is asymptotically stable conditionally to $\mathcal{A}$. Since $\Omega$ is asymptotically stable conditionally to $\mathcal{A}, \exists T=T(\epsilon)>$ $0 \mid d\left(\chi\left(T ; \chi_{0}\right), \Omega\right) \leq \frac{\epsilon}{2}$, with $\chi_{0} \in \mathcal{A}$. If we choose $\chi_{0}=$ $\chi(-T ; \boldsymbol{y}) \in \mathcal{A}$, then

$$
\frac{\epsilon}{2} \geq d\left(\chi\left(T ; \chi_{0}\right), \Omega\right)=d(\chi(0 ; \boldsymbol{y}), \Omega)=d(\boldsymbol{y}, \Omega)=\epsilon .
$$

Since this is a contradiction, we conclude that $\Omega$ must be stable.

Lemma 1. Let $V$ be a nonnegative function with $\dot{V}(\chi) \leq 0$, then $\mathcal{A}=\{\chi \mid V(\chi)=0\}$ is a positively invariant set and $\mathcal{A} \subset$ $E=\{\chi \mid \dot{V}(\chi)=0\}$.

Theorem 3. Let $\Omega$ be a bounded, invariant set for (26), and let $V(\chi)$ be a $C^{1}$ function such that $V(\chi) \geq 0, V(\Omega)=0$ and $\dot{V}(\chi) \leq 0$. If $\Omega$ is asymptotically stable conditionally to the largest positively invariant set $\mathcal{M}$ within $E=\{\chi \mid \dot{V}(\chi)=0\}$, then $\Omega$ is asymptotically stable.

Proof. In order to prove asymptotic stability we have to show stability and attractiveness.

From Lemma 1 it follows that $\mathcal{A}$ is a positively invariant set and $\mathcal{A} \subset E$, so since $\Omega$ is conditionally stable to $\mathcal{M}$ and $V(\Omega)=0$ i.e. $\Omega \subset \mathcal{A}$, then it must be conditionally stable to $\mathcal{A}$, hence by Theorem $2 \Omega$ is stable.

We will prove the attractiveness by contradiction. Since $\Omega$ is stable then $\forall \epsilon>0 \exists \delta=\delta(\epsilon)>0$ such that $\forall \chi_{0} \in B_{\delta}(\Omega) \Rightarrow$ $\chi\left(t ; \chi_{0}\right) \in B_{\epsilon}(\Omega), \forall t \geq 0$. Let $\mathrm{七}^{+}$be the positive limit set of the bounded solution $\chi\left(t ; \chi_{0}\right)$. Then $\mathrm{七}^{+}$is a positively invariant set and $\varkappa^{+} \in B_{\epsilon}(\Omega) \cap \mathcal{M}[30]$. Now let us assume by contradiction that $\mathrm{E}^{+}$is not $\Omega$. Since $\Omega$ is asymptotically stable conditionally to $\mathcal{M}$, then $\lim _{t \rightarrow \infty} d\left(\chi\left(t ; \chi_{0}\right), \Omega\right)=0$ if $\chi_{0} \in B_{\epsilon}(\Omega) \cap \mathcal{M}$. Choosing $\chi_{0}=y \in \mathrm{七}^{+} \neq \Omega$ we reach a contradiction.

\section{REFERENCES}

[1] A. De Luca, "Dynamic control of robots with joint elasticity," in IEEE Int. Conf. on Robotics and Automation (ICRA), Philadelphia, USA, Apr. 1988, pp. $152-158$.

[2] M. W. Spong and M. Vidyasagar, Robot Dynamics and Control. John Wiley and Sons, 1989.

[3] G. A. Pratt and M. M. Williamson, "Series elastic actuators," in IEEE/RSJ Int. Conf. on Intelligent Robots and Systems (IROS), Pittsburgh, USA, Aug. 1995, pp. 399-406.

[4] S. Wolf, O. Eiberger, and G. Hirzinger, "The DLR FSJ: Energy based design of a variable stiffness joint," in IEEE Int. Conf. on Robotics and Automation (ICRA), Shanghai, China, May 2011, pp. 5082-5089.

[5] G. Garofalo, C. Ott, and A. Albu-Schäffer, "Walking control of fully actuated robots based on the bipedal slip model," in IEEE Int. Conf. on Robotics and Automation (ICRA), Saint Paul, USA, May 2012, pp. 1999-2004.

[6] _ _ "Orbital stabilization of mechanical systems through semidefinite Lyapunov functions," in American Control Conference (ACC), Washington DC, USA, June 2013, pp. 5735-5741.

[7] G. Garofalo and C. Ott, "Limit cycle control using energy function regulation with friction compensation," IEEE Robotics and Automation Letters (RA-L), vol. 1, no. 1, pp. 90-97, 2016.

[8] T. McGeer, "Passive dynamic walking," Int. Journal of Robotics Research, vol. 9, pp. 62-82, 1990.
[9] H. Geyer, A. Seyfarth, and R. Blickhan, "Compliant leg behavior explains basic dynamics of walking and running," Proceedings of the Royal Society B, vol. 273, pp. 2861-2867, Nov. 2006.

[10] E. R. Westervelt, J. W. Grizzle, C. Chevallereau, J. H. Choi, and B. Morris, Feedback Control of Dynamic Bipedal Robot Locomotion. CRC Press, 2007.

[11] M. Garabini, A. Passaglia, F. Belo, P. Salaris, and A. Bicchi, "Optimality principles in variable stiffness control: The VSA Hammer," in IEEE/RSJ Int. Conf. on Intelligent Robots and Systems (IROS), San Francisco, USA, Sept. 2011, pp. 3770-3775.

[12] G. Garofalo, J. Englsberger, and C. Ott, "On the regulation of the energy of elastic joint robots: excitation and damping of oscillations," in American Control Conference (ACC), Chicago, USA, July 2015, pp. $4825-4831$.

[13] V. Duindam and S. Stramigioli, "Passive asymptotic curve tracking," in Proceedings of the IFAC Workshop on Lagrangian and Hamiltonian Methods for Nonlinear Control, Seville, Spain, Apr. 2003, pp. 229-234.

[14] _ - "Port-based asymptotic curve tracking for mechanical systems," European Journal of Control, vol. 10, pp. 411-420, 2004.

[15] M. Taniguchi and K. Fujimoto, "Asymptotic path following and velocity control of port-Hamiltonian systems," in European Control Conference (ECC), Budapest, Hungary, Aug. 2009, pp. $236-241$.

[16] C. Canudas-de-Wit, B. Espiau, and C. Urrea, "Orbital stabilization of underactuated mechanical systems," in Triennial World Congress of the International Federation of Automatic Control, Barcelona, Spain, July 2002, pp. 893-898.

[17] A. Shiriaev, J. W. Perram, and C. C. de Wit, "Constructive tool for orbital stabilization of underactuated nonlinear systems: Virtual constraints approach," IEEE Transactions on Automatic Control, vol. 50, no. 8, pp. 1164-1176, 2005.

[18] J. Park, W. Chung, and Y. Youm, "On dynamical decoupling of kinematically redundant manipulators," in IEEE/RSJ International Conference on Intelligent Robots and Systems, 1999, pp. $1495-1500$.

[19] C. Ott, Cartesian Impedance Control of Redundant and Flexible-Joint Robots, ser. Springer Tracts in Advanced Robotics. Berlin: SpringerVerlag, 2008.

[20] C. Ott, A. Kugi, and Y. Nakamura, "Resolving the problem of nonintegrability of nullspace velocities for compliance control of redundant manipulators by using semi-definite Lyapunov functions," in IEEE Int. Conf. on Robotics and Automation (ICRA), Pasadena, USA, May 2008, pp. $1456-1463$.

[21] A. Albu-Schäffer, C. Ott, and F. Petit, "Energy shaping control for a class of underactuated Euler-Lagrange systems," in Proc. 10th Int. IFAC Symp. Robot Control, Dubrovnik, Croatia, Sept. 2012, pp. 567-575.

[22] M. W. Spong, "Modeling and control of elastic joint robots," the ASME Journal of Dynamic Systems, Measurement, and Control, vol. 109, pp. $310-318,1987$.

[23] C. Canudas-de-Wit, B. Siciliano, and G. Bastin, Theory of robot control. London: Springer-Verlag, 1996.

[24] T. Frankel, The Geometry of Physics: An Introduction. New York: Cambridge University Press, 2004.

[25] B. Siciliano, L. Sciavicco, L. Villani, and G. Oriolo, Robotics: Modelling, Planning and Control. Springer Publishing Company, Incorporated, 2008.

[26] J. Baillieul, "Kinematic programming alternatives for redundant manipulators," in IEEE Int. Conf. on Robotics and Automation (ICRA), St. Louis, USA, Mar. 1985, pp. 722-728.

[27] G. Garofalo, C. Ott, and A. Albu-Schäffer, "On the closed form computation of the dynamic matrices and their differentiations," in IEEE/RSJ Int. Conf. on Intelligent Robots and Systems (IROS), Tokyo, Japan, Nov. 2013, pp. 2364-2359.

[28] A. Iggidr, B. Kalitine, and R. Outbib, "Semidefinite Lyapunov functions: Stability and stabilization," in Mathematics of Control, Signals, and Systems, 1996, pp. 95-106.

[29] R. Sepulchre, M. Jankovic, and P. Kokotovic, Constructive Nonlinear Control. Berlin: Springer, 1997.

[30] H. K. Khalil, Nonlinear Systems. New Jersey: Prentice Hall, 2002. 